EXTENDED REPORT

\title{
Acute zonal occult outer retinopathy: towards a set of diagnostic criteria
}

\author{
P J Francis, A Marinescu, F W Fitzke, A C Bird, G E Holder
}

Br J Ophthalmol 2005;89:70-73. doi: 10.1136/bjo.2004.042416

See end of article for authors' affiliations

.....................

Correspondence to: Graham E Holder, $\mathrm{PhD}$, Department of Electrophysiology, Moorfields Eye Hospital, 162 City Road, London ECIV 2PD, UK; Graham Holder@moorfields.nhs.uk

Accepted for publication 5 May 2004
Background: Individuals with acute zonal occult outer retinopathy (AZOOR) present with initially progressive scotomata and photopsia. Characteristically, the extent of the visual field defect is unexplained by fundal examination, but there is marked retinal dysfunction evident electrophysiologically. It is the authors' experience that a group of patients exhibit characteristic clinical and electrophysiological abnormalities, which serve as criteria for a working diagnosis.

Methods: A retrospective observational case series of 28 patients were identified with the clinical diagnosis of AZOOR who shared similar abnormal electrophysiology. Details of the history and ophthalmic findings were obtained from the case notes.

Results: Electrophysiology demonstrated a consistent pattern of dysfunction both at the photoreceptor/ retinal pigment epithelial complex but also at inner retinal levels, essentially comprising a delayed $30 \mathrm{~Hz}$ flicker ERG and a reduction in the EOG light rise.

Conclusion: This study determines diagnostic criteria applicable to a group of patients with AZOOR, typically those with classic symptomatology. Electrophysiological testing can help avoid lengthy, costly, and potentially invasive investigations, and the unnecessary use of immunosuppressive therapy.
A decade has passed since Gass ${ }^{1}$ introduced the acronym AZOOR (acute zonal occult outer retinopathy) to describe a previously unrecognised syndrome occurring predominantly in young white females. In his original series of 13 patients, affected individuals typically presented with central photopsia associated with progressive scotomata and blurring of vision. Strikingly, fundal examination was unremarkable despite marked retinal dysfunction detectable electrophysiologically. After several months, the condition usually stabilised with later development of retinal pigment epithelial atrophy and intraretinal pigment migration corresponding to the zones of field loss. Bilateral involvement was frequent. Although a viral or autoimmune aetiology was suspected, no cause was readily identifiable in this group of generally healthy patients.

Subsequent reports ${ }^{2-4}$ have appeared to confirm Gass's initial suspicion that there is considerable coincidence between AZOOR and the so called "white dot" syndromes -namely, MEWDS (multiple evanescent white dots syndrome), AIBSE (acute idiopathic blind spot enlargement syndrome), AMN (acute macular neuroretinitis), PIC (punctate inner choroiditis), MIC (multifocal inner choroiditis), MCP (multifocal choroiditis with panuveitis), and P-POHS (pseudo-presumed ocular histoplasmosis syndrome). All these conditions present predominantly in young females and in certain instances are accompanied by unexplained visual field loss and similar electrophysiological abnormalities. ${ }^{5}$ Indeed, it is not clear if AZOOR should be considered part of this spectrum of disorders; whether the "white dot" syndromes are manifestations of or may precipitate AZOOR; or even whether they share a similar aetiology. ${ }^{6}$ AAOR (acute annular outer retinopathy) may be a variant of AZOOR. ${ }^{7}$

It has become our experience that a group of our patients suspected of AZOOR, in particular those whose symptoms most closely parallel those of Gass's original cohort, exhibit characteristic electrophysiological abnormalities. The goal of this article is therefore to define the clinical and electrophysiological characteristics in these patients.

\section{PATIENTS AND METHODS}

All patients were identified retrospectively from the entire cohort of patients undergoing electrophysiological testing with the clinical suspicion of AZOOR. The department is a tertiary referral centre, and the case mix was therefore drawn not only from the local patient catchment area but also included a significant number of regional and supraregional referrals.

The history and results of clinical examination were obtained from the case notes. All individuals underwent electrophysiology performed to incorporate and exceed the ISCEV standards for EOG, ${ }^{8}$ pattern (PERG), ${ }^{9}$ and full field ERG.$^{10}$ In addition, photopic ON/OFF responses ${ }^{11}$ and $S$ cone specific ERGs ${ }^{12}$ were assessed in the majority of patients.

\section{Statistical analysis}

Data obtained were non-parametrically distributed and are thus expressed in terms of median, range, and 25th-75th interquartile range compared to our normal laboratory range (range $+5 \%$ ).

\section{RESULTS}

\section{Patient characteristics}

Twenty eight patients were identified in whom there was a consistent pattern of electrophysiological abnormality. Most were young, generally healthy females (females 24 (86\%), males four (14\%); median age 43 , range $23-75$ years) who presented with blurred vision (median best corrected acuity $6 / 12$, range $6 / 6$ to hand movements). In all but one individual, one or more zones of acute visual field loss were documented by automated static threshold perimetry.

Abbreviations: AIBSE, acute idiopathic blind spot enlargement syndrome; $A M N$, acute macular neuroretinitis; $A Z O O R$, acute zonal occult outer retinopathy; EOG, electro-oculography; ERG,

electroretinogram; MCP, multifocal choroiditis with panuveitis; MEWDS, multiple evanescent white dots syndrome; MIC, multifocal inner choroiditis; PERG, pattern ERG; PIC, punctate inner choroiditis; P-POHS, pseudo-presumed ocular histoplasmosis syndrome 
A
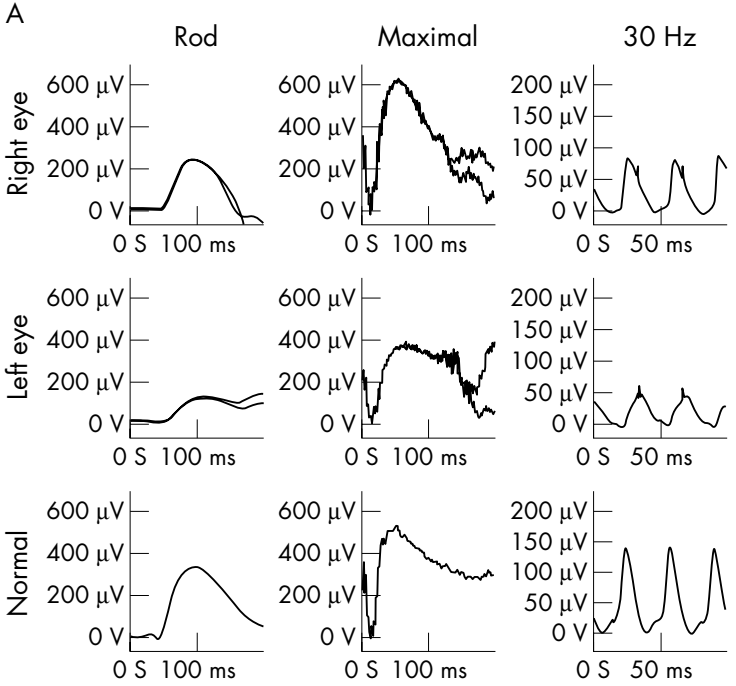

$200 \mu \mathrm{V}$

$150 \mu \mathrm{V}$

$100 \mu \mathrm{V}$

$50 \mu \mathrm{V}$
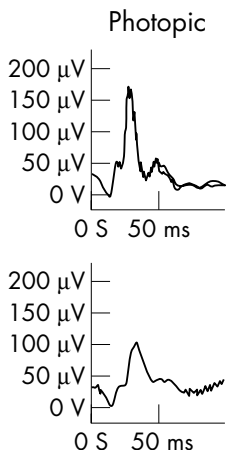

$200 \mu \mathrm{V}-$

$150 \mu \mathrm{V}$

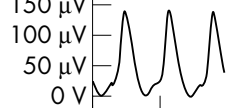

$0 S 50 \mathrm{~ms}$

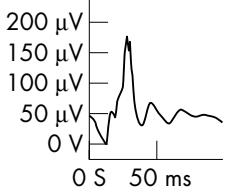

Figure 1 Electrophysiological abnormalities characteristic of eyes with AZOOR. Left eye rod specific ERG is

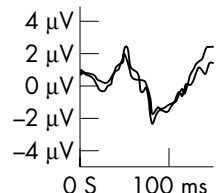
inormal; maximal response shows both a-wave and b-wave amplitude reduction; $30 \mathrm{~Hz}$ flicker ERG is markedly delayed and mildly subnormal; photopic single flash b-

$100 \mathrm{~ms}$ wave amplitude is subnormal; PERG $4 \mu \mathrm{V}-\quad$ P50 is subnormal in keeping with $2 \mu \mathrm{V}-\quad$ show no definite abnormality. The EOG $0 \mu \mathrm{V}$ ard $\sqrt{\sim}$ light rise is markedly reduced from the $-2 \mu \mathrm{V}-\mathrm{a}$ left eye compared with that from the

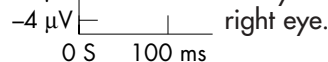

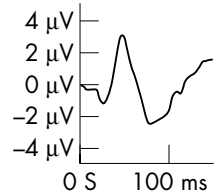

B

Right eye

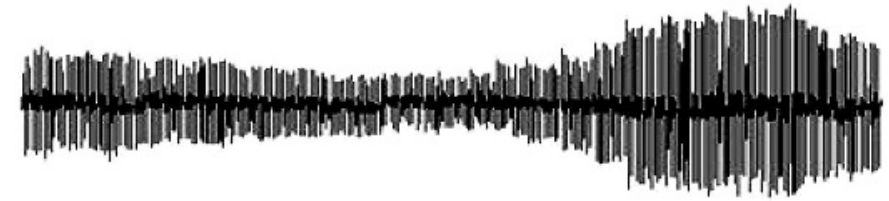

Left eye

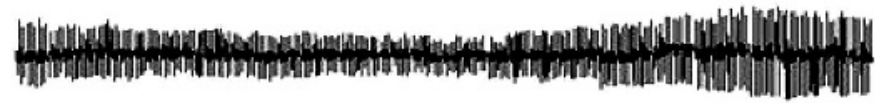

Scotomata were most frequently temporal and complete, initially progressive, then persistent, with photopsia typically projected into the major zone of field loss. Fifteen patients (54\%) had unilateral symptoms and 13 (46\%) bilateral symptoms at presentation. Four initially unilateral cases (14\%) subsequently developed symptoms suggestive of AZOOR in the fellow eye.

\section{Relevant ophthalmic findings}

Eight patients were myopic. In 13 individuals (46\%) there was a previous history of one of the "white dot" syndromes, most frequently MEWDS or PIC. Otherwise, no relevant fundal abnormalities were seen in the early stages of the disease. In those six patients where long term follow up data were available and in whom no evidence of a "white dot" syndrome was present, two were noted to develop a diffuse outer pigmentary retinopathy.

\section{Electrophysiology}

Five patients $(18 \%)$ with unilateral symptomatology were found to have electrophysiological evidence suggestive of bilateral dysfunction. These were considered for the purposes of analysis as bilateral cases. Data are thus presented on 46 eyes.

The characteristic pattern of electrophysiological abnormality is shown in figure 1. Despite the suspicion of only focal retinal involvement symptomatically, and often no fundal abnormality, there was evidence of generalised dysfunction affecting not only the cone system, but also the retinal pigment epithelium as shown by a marked reduction in the light rise of the EOG in the absence of a comparable degree of rod ERG abnormality. The EOG light rise was consistently abnormal; either abolished, subnormal or showing significant relative reduction compared with the fellow eye. The P50

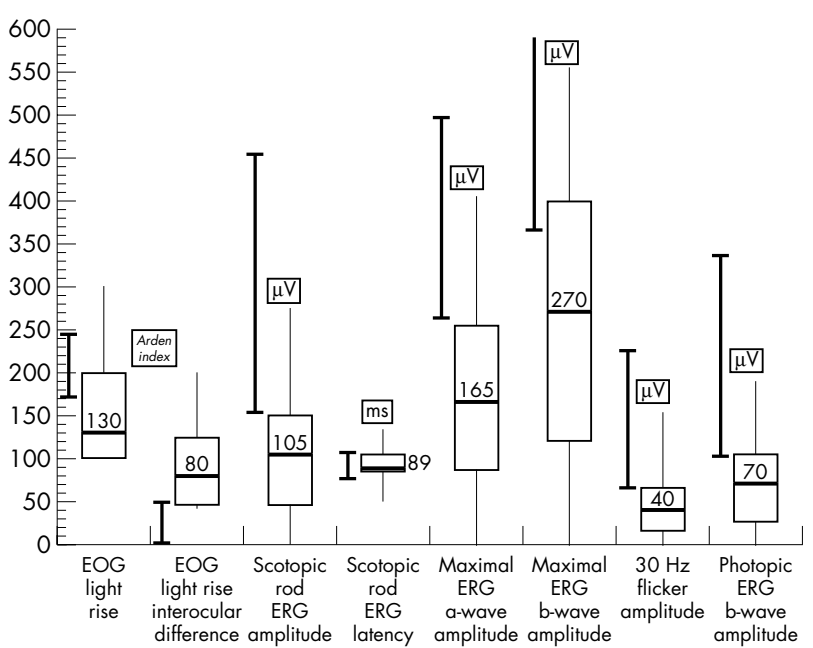

Figure 2 Box and whisker plots of electrophysiological abnormalities expressed as microvolts $(\mu \mathrm{V})$ and milliseconds (ms) where appropriate. Closed bars show range $+5 \%$ of normal electrophysiological responses in our laboratory control population. Open box shows 25th-75th interquartile range with the overall range encompassed by the whiskers. The median value is shown by the horizontal bar within box. PERG P50, pattern ERG P50.

component of the PERG of affected eyes was reduced in amplitude with preservation of the P50:N95 ratio in keeping with macular dysfunction. ${ }^{13}$ In the full field ERGs, rod specific ERG amplitudes were often reduced without latency change; standard ERG a-wave and b- wave amplitudes were reduced without latency change; and oscillatory potentials, related to amacrine cell function, were attenuated. In all 
affected eyes, the $30 \mathrm{~Hz}$ cone flicker response showed implicit time delay, often with associated amplitude reduction. ON and OFF responses, when recorded, were of increased latency; S-cone responses were reduced or poorly formed. Quantification of the anomalies is summarised in table 1 and figures 2 and 3.

\section{DISCUSSION}

Acute zonal occult outer retinopathy (AZOOR) is currently a diagnosis made entirely on clinical grounds and is frequently a diagnosis of exclusion. ${ }^{6}$ Our cases have features in common with those of previous reports, ${ }^{14}$ but in particular with Gass's original cohort of patients. ${ }^{1}$ They were mostly relatively young and healthy females presenting with central photopsia or shimmering; had unilateral acute or subacute visual field loss that could not be explained by the fundal changes; there was reduced visual acuity and usually a unilateral presentation. Subsequent involvement of the fellow eye was observed in some individuals.

The cases are also defined by a characteristic pattern of electrophysiological abnormality. In particular, the most consistent abnormalities were a delay in the cone system derived $30 \mathrm{~Hz}$ flicker response and a reduction in the light rise of the electro-oculogram. Standard ERG response a-wave amplitudes could be reduced in keeping with photoreceptor dysfunction, but the magnitude of this reduction was not sufficient to account for the reduction in EOG. Where cone bipolar cell function was assessed with ON and OFF response recording, prolongation of $b$-wave implicit times indicated ON pathway involvement. The PERG P50 was reduced in all affected eyes.

Jacobson $e t \mathrm{al}^{2}$ reported the electrophysiological findings in 24 patients with MEWDS, AIBSE, AMN, and P-POHS which they at the time grouped under the name AZOOR. Common to their patients were the additional complaints of rapidly progressive field defects and photopsia making them similar to our series and consistent with current categorisation. ${ }^{14}$ Their results were in agreement with previous reports ${ }^{15}$ suggesting that patients with AZOOR showed a pattern of visual dysfunction that was photoreceptor in origin. EOGs were not performed.

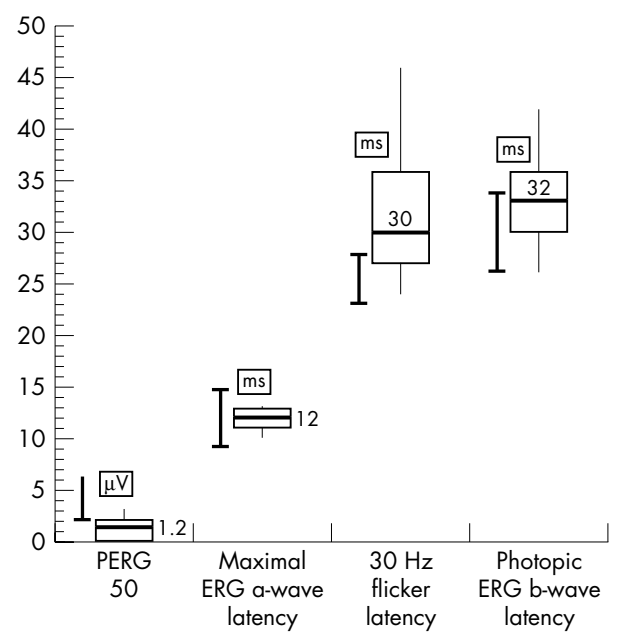

Figure 3 Box and whisker plots of electrophysiological abnormalities expressed as microvolts $(\mu \mathrm{V})$ and milliseconds (ms) where appropriate. Closed bars show range $+5 \%$ of normal electrophysiological responses in our laboratory control population. Open box shows 25th-75th interquartile range with the overall range encompassed by the whiskers. The median value is shown by the horizontal bar within box. PERG P50, pattern ERG P50.
Our results provide significant refinement of previous electrophysiological data. We confirm dysfunction at the retinal pigment epithelium/photoreceptor complex and show that cones appear more affected than rods. However, the $30 \mathrm{~Hz}$ flicker ERG derives from the inner retina ${ }^{15}$ and thus calls into question whether AZOOR is indeed exclusively an outer retinopathy. Given the focal nature of the disease clinically, it is fascinating that electrophysiological dysfunction appears global. Such a pattern is not observed in other disorders. In particular, the "white dot syndromes" (when not associated with the subsequent development of AZOOR) or other inflammatory diseases of the retina or choroid typically show either no or only focal electrophysiological abnormality. ${ }^{16}$ Further, the reduction in the EOG light rise is not a characteristic finding in those conditions.

The cause of AZOOR remains unknown though several disease mechanisms have been suggested. The electrophysiological abnormalities prevailing in our cases, in which prolongation of implicit times was disproportionately greater than amplitude reductions, favour an inflammatory aetiology. ${ }^{13}$ Disorders such as MEWDS, PIC, and macular neuroretinitis usually give restricted loss of function and thus are not routinely associated with an abnormal full field ERG; the presence of an abnormal full field ERG with implicit time shift signals generalised retinal dysfunction. Where inflammatory disease leads to generalised retinal dysfunction, for example birdshot chorioretinopathy, the $30 \mathrm{~Hz}$ flicker ERG is frequently delayed but may be of normal amplitude.

The lack of causative ophthalmoscopic abnormalities in our series implies that cell death does not occur early in the disease as would be expected with an infective aetiology such as a virally mediated retinitis. The pigmentary changes seen late in disease would thus be consistent with death of previously damaged or targeted cells followed by intraretinal pigment migration as occurs in inherited retinal degenerations.

Given that approximately half our patients had suffered a preceding episode of MEWDS or PIC, it is tempting to propose that AZOOR is an autoimmune mediated disorder triggered by an as yet unascertained process precipitated by the previous inflammatory event. The transient nature of the diagnostic fundus abnormality in MEWDS may explain why many patients with AZOOR have normal fundi since there is often a delay between the onset of symptoms and the diagnosis of AZOOR. Increasing use of indocyanine green angiography may provide data that enable this hypothesis to be examined. It should be stressed that most patients with PIC, MEWDS, etc, do not proceed to develop AZOOR, but it cannot be excluded that MEWDS or PIC may be a limited form of AZOOR. The clinical features of these patients closely resemble the initial description of AZOOR, ${ }^{1}$ but our data do not support the later extension ${ }^{6}$ to include other conditions that might share a similar aetiology.

\section{CONCLUSIONS}

We have demonstrated that there is a group of patients with the diagnosis of AZOOR in whom there is a distinctive pattern of clinical and electrophysiological abnormality. These provide a set of diagnostic criteria which clinically resemble those initially described by Gass. ${ }^{1}$ The patient presents with photopsia and a central field defect that is unexplained by any abnormality of the fundus. There is a delayed $30 \mathrm{~Hz}$ flicker ERG and a reduction in the EOG light rise. It is possible that the group of disorders such as MEWDS, PIC, MIC, macular neuroretinitis, AIBSE, etc, may be precipitating factors, but most patients with those disorders do not proceed to develop AZOOR. The importance of electrophysiological assessment in establishing the diagnosis is evident. Interestingly, although the clinical features of these patients closely resemble the initial description of 
Table 1 Electrophysiological abnormalities in patients with AZOOR

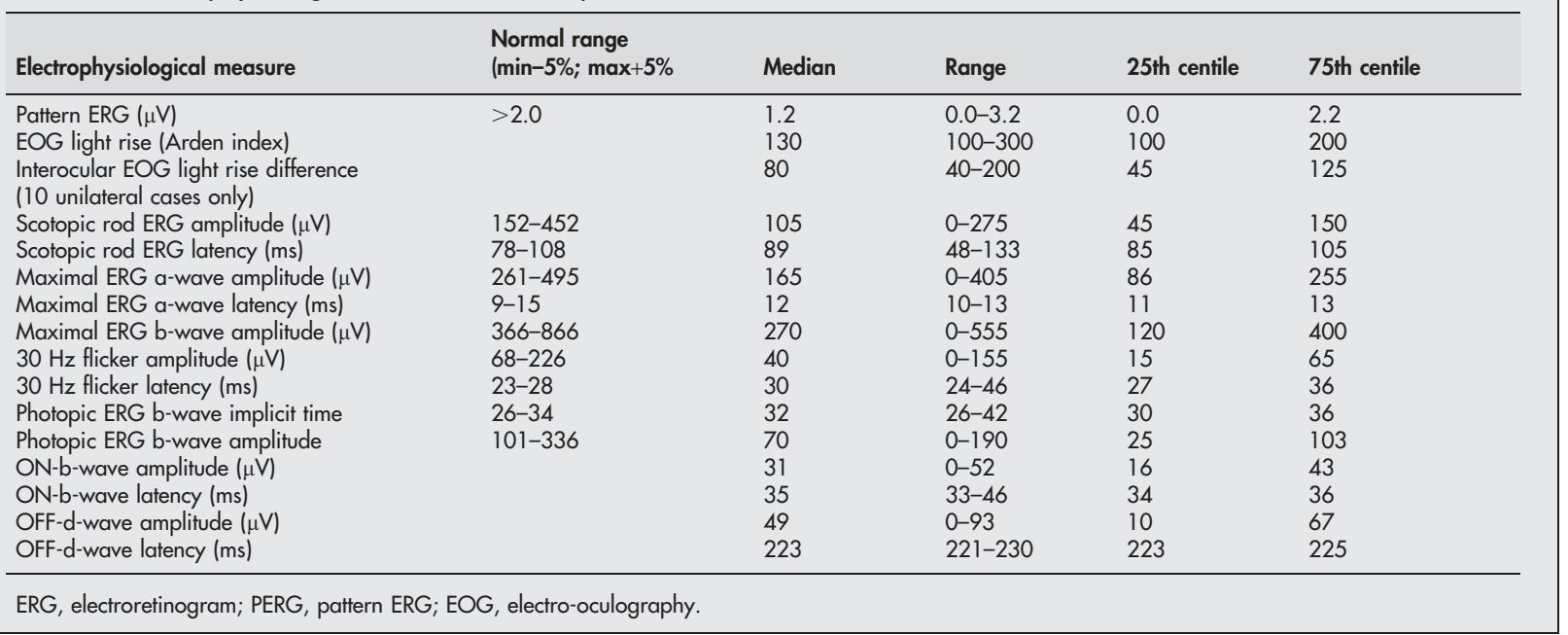

AZOOR $^{1}$ our data do not support the later extension, ${ }^{6}$ to include other conditions that might share a similar aetiology.

This study serves to alert the physician to consider the diagnosis of AZOOR in individuals presenting with scotomata unexplained by ocular examination, particularly where central photopsia are a prominent feature. We suggest that our study has demonstrated a characteristic pattern of clinical and electrophysiological abnormality that establishes the diagnosis thus avoiding lengthy, costly and invasive neurological and systemic investigations and the unnecessary use of immunosuppressive agents. The possible role of the "white dot" syndromes as precipitating factors requires further investigation.

\section{Authors' affiliations}

P J Francis, F W Fitzke, A C Bird, Institute of Ophthalmology, UCL, 11 43 Bath Street, London, UK

P J Francis, A Marinescu, A C Bird, G E Holder, Moorfields Eye Hospital, 162 City Road, London, UK

\section{REFERENCES}

1 Gass J. Acute zonal occult outer retinopathy. J Clin Neuro Ophthalmol 1993;13:79-97.

2 Jacobson S, Morales D, Sun X, et al. Pattern of retinal dysfunction in acute zonal occult outer retinopathy. Ophthalmology 1995; 102:1 187-96.
3 Volpe N, Rizzo J, Lessell S. Acute idiopathic blind spot enlargement syndrome. Arch Ophthalmol 2001;119:59-63.

4 Holz F, Kim R, Schwartz S, et al. Acute zonal occult outer retinopathy (AZOOR) associated with multifocal choroidopathy. Eye 1994;8:77-83.

5 Nakao K, Isashiki M. Multiple evanescent white dot syndrome. Jpn J Ophthalmol 1986;30:376-84.

6 Gass J. The acute zonal outer retinopathies. Am J Ophthalmol 2000;130:655-7.

7 Fekrat S, Wilkinson C, Chang B, et al. Acute annular outer retinopathy: report of four cases. Am J Ophthalmol 2000;130:636-44.

8 Marmor MF, Zrenner E. Standard for clinical electro-oculography. International Society for Clinical Electrophysiology of Vision. Arch Ophthalmol 1993;111:601-4.

9 Bach M, Hawlina M, Holder G, et al. Standard for pattern electrophysiology. International Society for Clinical Electrophysiology of Vision. Doc Ophthalmol 2000;101:11-18.

10 Marmor MF, Zrenner E. Standard for clinical electroretinography (1999 update). International Society for Clinical Electrophysiology of Vision. Doc Ophthalmol 1998;97:143-56.

11 Sieving P. Photopic ON- and OFF-pathway abnormalities in retinal dystrophies. Trans Am Ophthalmol Soc 1993:91:701-73.

12 Arden G, Wolf J, Berninger T, et al. S-cone ERGs elicited by a simple technique in normals and in tritanopes. Vis Res 1999;39:641-50.

13 Holder GE. Pattern electroretinography (PERG) and an integrated approach to visual pathway diagnosis. Prog Retina Eye Res 2001;20:531-61.

14 Gass J, Agarwal A, Scott I. Acute zonal occult outer retinopathy: a long term follow-up study. Am J Ophthalmol 2002;134:329-39.

15 Sieving P, Fishmann G, Jampol L, et al. Multiple evanescent white dot syndrome: II. Electrophysiology of the photoreceptors during retinal pigment epithelial disease. Arch Ophthalmol 1984;102:675-9.

16 Huang HJ, Yamazaki H, Kawabata H, et al. Multifocal electroretinogram in multiple evanescent white dot syndrome. Doc Ophthalmol 1996;97/92: 301-9. 